\title{
Role of Circular RNA in Melanoma Development: A Mini Review
}

\author{
C. ZHIBING, C. JUNJIE ${ }^{1}$, W. QINGBIAO, H. QIANG, FENG SHUANG, HE MEI, XIAOHAN CHEN²* AND CEN YING \\ Department of Cosmetic Plastic Surgery, Chengdu Second People's Hospital, Chengdu, Sichuan, China , ${ }^{1}$ Department of \\ Cosmetic Plastic and Burn Surgery, West China Hospital Sichuan University, Chengdu, Sichuan, China , ${ }^{2}$ Division of Geriatrics, \\ Sichuan Academy of Medical Sciences and Sichuan Provincial People's Hospital, Chengdu, China
}

\section{Zhibing et al.: Role of Circular RNA in Melanoma Development}

\begin{abstract}
The Malignant melanoma, a tumor derived from melanocytes, shows severe drug resistance and prompt metastasis, causing a serious threat to human health. Emerging evidence indicates that circular RNAs are widely expressed in mammals and play crucial role in tumorigenesis by regulating micro RNA, downstream gene expression and protein production. The role of circular RNA in pathogenesis of melanoma has been recently reported and highlighted. This review makes and attempt to provide a better insight in to the role of circular RNA in melanoma.
\end{abstract}

Key words: Melanoma, circRNA, melanocytes, mutations

Melanoma is an aggressive cancer developed from melanocytes found predominantly in the skin, but also in the eyes, ears, gastrointestinal tract and oral and genital mucosal membranes. The global incidence of melanoma has increased dramatically over the past few decades. Furthermore, melanoma is a leading cause of skin cancer deaths in the United States ${ }^{[1]}$, which is also one of the most challenging cancers to diagnose and treat because it requires high levels of expertise to detect the lesions at an early stage. Melanoma survival rates are good when the disease is detected early and treated by surgical resection. Invasive melanoma cells escape from their primary location and translocate via the circulatory system or body cavities to lymph nodes or distant organs to establish a secondary cancer tissue. The underlying molecular biology of melanomas is complex and involves interactions between melanoma cells and components of the microenvironment ${ }^{[2,3]}$, which has a very high frequency of acquired mutations that are critically involved in proliferation, survival, and metastasis ${ }^{[3,4]}$. Emerging evidence has indicated that genetic and epigenetic factors have an important impact on the progress of melanoma. In recent years, the expansion of knowledge of non-coding RNA (ncRNA) biology has promoted the comprehensive understanding of the critical roles of ncRNA in tumorigenic processes ${ }^{[5-8]}$. Circular RNAs (circRNAs) are an emerging class of primarily ncRNAs, which arise by cis back splicing and cyclization of portions of protein-coding genes and other transcripts. With the development of high-throughput sequencing technology and bioinformatics, the significance of circRNAs in cancer development have been revealed gradually. Concerning important pathway, the novel pathological mechanism of these circRNAs in melanoma development have been elucidated over the last $2 \mathrm{y}$, the emerging roles of circRNA in melanoma pathophysiology and regulation were discussed in this review.

CircRNA, which is a covalent closed loop structure, with neither $5^{\prime}$ to $3^{\prime}$ polarity nor polyadenosine (A) tail, is widely distributed in mammals, especially in humans and mice and plays an important role in regulating gene expression and various cellular processes ${ }^{[9]}$. In molecular biology, circRNA as microRNA (miRNA) sponges are involved in regulating miRNA functions and expression of downstream target genes of miRNA by combining corresponding miRNA binding sites ${ }^{[10]}$. miRNA are epigenetic factors that control amount processes including cell proliferation and differentiation, cell senescence, survival, autophagy and migration and contribute to changes in cellular metabolism and genome stability. Therefore, even slight alterations in miRNA levels can lead to a variety of pathologies, including cancer ${ }^{[11-15]}$. 
The expression patterns of circRNA in melanoma were investigated in a few studies. Several differentially expressed circRNAs in melanoma were revealed. $\mathrm{Ju}$ et al. reported that 90 circRNAs were significantly dysregulated in the metastatic oral mucosal melanoma when compared to the paired adjacent tissues, among which 58 were upregulated and 32 were downregulated ${ }^{[16]}$. Wang et al. reported that 797 circRNAs were upregulated and 969 circRNAs were downregulated in the low-metastatic melanoma cell line, WM35 cells, while 307 circRNAs were upregulated and 312 circRNAs were downregulated in the high-metastatic melanoma cell line WM451 cells $^{[17]}$. Yang et al. reported that 50579 circRNAs were identified as being dysregulated in uveal melanoma. Among them, 20654 were upregulated and 29925 were downregulated in uveal melanoma samples compared with normal uveal samples ${ }^{[18]}$.

Some studies have found that circRNAs can predict the prognosis of melanoma patients. High expression of circ_0025039 was reported to predict a poor prognosis in melanoma patients. Circ_0025039 expression was significantly related to the pathological node status, pathological metastasis status and clinical stage. Melanoma patients with high expression of circ_0025039 were found to have a shorter survival time than those with low expression of circ_0025039, suggesting that high expression of circ_0025039 was associated with a poor prognosis ${ }^{[19]}$. CircRNA_0084043 was identified to be significantly upregulated in melanoma and was an independent risk factor of overall survival for melanoma patients. In this study, the high circRNA_0084043 expression group had a poorer survival. Univariate and Multivariate Cox regression analysis further demonstrated that high circRNA_0084043 expression was an independent risk factor of overall survival for melanoma patients ${ }^{[20]}$.

CircRNAs were identified to play a critical role in melanoma growth and metastasis circ_0084043 was found to play an oncogenic role of melanoma. Circ_0084043 knockdown could significantly restrain the tumor growth as evidenced by reduced tumor volume and tumor weight in melanoma xenograftmice ${ }^{[21]}$. Also, silencing circ_0025039 was demonstrated to decrease the melanoma volumes and weight in $v i v o^{[19]}$. Additionally, it was also found that circRNAs, which are abundant in eukaryotic cells, especially in mammalian brains are reported to be associated with human neurodegenerative diseases and also silencing of circ_0025039 was revealed to decrease the tumor volumes and weight. Furthermore, CDR1as was demonstrated to alter melanoma metastasis in vivo. Enhanced melanoma lung metastasis in mice bearing tumors depleted of CDR1as was observed ${ }^{[22]}$.

The molecular and physiological functions of most circRNAs remain unknown, but there has been increasing progress in this area as a result of the development of methods to overexpress and knock-down/out specific circRNAs. The data revealed that knockdown of circ_0084043 could suppress melanoma proliferation, migration and invasion, but promote apoptosis in A375 and SK-MEL-28 melanoma cells, indicating an oncogenic role of circ 0084043 in the development of melanoma ${ }^{[21]}$. Silencing of circMYC inhibited the growth rate and viability of Mel-CV melanoma cells. As expected overexpression of circMYCdemonstrated the opposite effects on Mel-CV cells. Therefore, upregulation of circMYC can promote the melanoma cell growth ${ }^{[23]}$. Also, silencing of circ_0025039 inhibited melanoma cell proliferation and invasion. The assays revealed that the melanoma cell proliferation and invasion abilities were significantly decreased in the si-circ_0025039 group compared with those in the negative control (NC) group ${ }^{[19]}$. Additionally, silencing of circ0000082 and circ0016418 were demonstrated to inhibit the proliferation and decrease the invasion capability of the WM451 melanoma cells ${ }^{[17]}$. The data demonstrated that cell proliferation, migration, invasion, and epithelial-mesenchymal transition were all restrained in skin melanoma cells after circ0016418 was knocked down ${ }^{[24]}$. However, circ0023988, circ0008157 and circ0030388 were demonstrated to suppress melanoma invasion. The invasion ability of low-metastatic melanoma WM35 cells was found to be enhanced by silencing of circ0023988, circ0008157 and circ0030388 $8^{[17]}$.

Novel data supported that circRNAs were involved in glycometabolism and lactate production. The glucose consumption rate was significantly decreased in the si-circ_0025039 group compared with that in the NC group, and the lactate production rate was also lower in the si-circ_0025039 group than that in the NC group ${ }^{[19]}$. Additionally, circMYC was found to promote glycolysis and lactate production ${ }^{[23]}$. Silencing of circMYC significantly decreased extracellular acidification rate (ECAR) in Mel-CV cells, while the forced expression of circMYC significantly augmented ECAR. Consistent with the effects of circMYC on glucose uptake, extracellular lactate production significantly decreased after knockdown circMYC while the application of over 
TABLE 1: circRNAs EITHER UPREGULATED OR DOWNREGULATED IN MELANOMA

\begin{tabular}{|c|c|c|c|c|}
\hline CirRNA & Target miRNA & Downstream regulation Mechanism & Type of experiment & References \\
\hline \multicolumn{5}{|l|}{ Downregulated } \\
\hline CDR1as & $\operatorname{miR}-7$ & EGFR, IGF-1, IRS-2, PAX6, CPAR & in vitro & {$[26]$} \\
\hline $\begin{array}{l}\text { Upregulated } \\
\text { CircMTUS1 }\end{array}$ & miR-622, miR-1208 & $\begin{array}{l}\text { ErbBsignaling pathway, } \\
\text { MAPK signaling pathway, } \\
\text { Wntsignaling pathway, }\end{array}$ & $\begin{array}{l}\text { in vitro } \\
\text { in vivo }\end{array}$ & [27] \\
\hline circMYC & $\operatorname{miR}-1236$ & LDHA activity & $\begin{array}{l}\text { Human tissue } \\
\text { in vitro }\end{array}$ & [23] \\
\hline Circ_0016418 & miRNA-625 & Yin Yang 1 & $\begin{array}{l}\text { Human tissue } \\
\text { in vitro }\end{array}$ & [24] \\
\hline circ_0025039, & miR-198 & CDK4 & $\begin{array}{l}\text { Human tissue } \\
\text { in vitro } \\
\text { in vivo }\end{array}$ & [19] \\
\hline circ_0084043 & $\operatorname{miR}-153-3 p$ & Snail & $\begin{array}{l}\text { Human tissue } \\
\text { in vitro }\end{array}$ & {$[20]$} \\
\hline circ_0084043 & $\operatorname{miR}-429$ & $\begin{array}{l}\text { Wnt/B-catenin pathway, } \\
\text { tribbles homolog } 2\end{array}$ & $\begin{array}{l}\text { Human tissue } \\
\text { in vitro } \\
\text { in vivo }\end{array}$ & [28] \\
\hline
\end{tabular}

expression of circMYC generated opposite effects ${ }^{[23]}$. Circ_ITCH over expression was reported to inhibit glucose uptake in melanoma cells by down regulating GLUT1 ${ }^{[25]}$. Comparing Control (C) and NC, expression levels of circ_ITCH and GLUT1 were significantly upregulated in those cells at $24 \mathrm{~h}$ after transfections. Therefore, circ_ITCH overexpression significantly suppressed glucose uptake in melanoma cells ${ }^{[25]}$.

The recent reports indicated that circRNAs could act as miRNA sponges to bind functional miRNAs and liberate mRNA transcripts targeted by miRNAs. Seven circRNAs were found to have possible target miRNAs in melanoma. Zhang et al. reported that CDR1as, as a mir7 sponge, was predicted to be important in regulating melanoma development ${ }^{[26]}$. However, Hanniford et al. have revealed that $\mathrm{CDR} 1$ as promoted melanoma invasion in vitro and metastasis in vivo through a miR7-independent, IGF2BP3-mediated mechanism ${ }^{[22]}$. Shang et al. have reported that Silencing of circMTUS1 inhibited conjunctival melanoma proliferation in vitro and in vivo. CircMTUS1 may serve as an oncogene by binding to hsa-miR-622 and hsa-miR-1208 to regulate several tumor-related pathways, such as ErbB, MAPK and Wntsignallingpathways ${ }^{[27]}$. Jin et al. have shown that circMYC directly bound to miR-1236 as a molecular sponge that targeting miR-1236 in Mel-CV cells and 3' UTR of LDHA acted as a target of miR1236 using Mel-CV cells ${ }^{[23]}$. Zou et al. have found that miR-625 expression was boosted by the knockdown of circ0016418. Overexpression of miR-625 was evident and miR-625 significantly reduced the YY1 mRNA and protein levels. Thus, circ0016418 negatively regulated the miR-625 level and YY1 was a target of miR-625 ${ }^{[24]}$ Additionally, Bian et al. have observed that circ_0025039 markedly inhibited the luciferase intensity of miR-198 and silencing of circ_0025039 significantly upregulated miR-198 expression ${ }^{[19]}$. Luan et al. have reported that circRNA_0084043 was reported to promote melanoma cell growth and metastasis by sponging miR-153-3p and upregulating Snail expression. Interestingly, circRNA_0084043 was also found to promote melanoma cell proliferation, migration and invasion and tumor growth, through sponging miR-429 and up regulating tribbles homolog $2^{[28]}$. All the above data indicate that circRNAs as important miRNA sponges regulate melanoma development (Table 1).

Despite recent advances in cancer treatment, malignant melanoma remains the main cause of skin cancer deaths. The findings reviewed here indicate the involvement of circRNAs in melanoma development. Considering the role of circRNAs in melanoma development, circRNAs or their antagonists may be considered for potential therapeutic applications, however, the strategies for this approach are still under development. Certain altered expression of circRNAs has been found to correlate with poor prognosis of melanoma. Additional studies are needed to identify more cirRNAs, which could predict the prognosis of melanoma or response to treatment. Therefore, CircRNAs, may be useful as biomarkers for early melanoma detection.

\section{Conflict of interest:}

The authors report no conflicts of interest in this work.

\section{REFERENCES}

1. Siegel R, Ma J, Zou Z, Jemal A. Cancer statistics, 2014. CA Cancer J Clin 2014;64:9-29. 
2. Adler NR, Haydon A, McLean CA, Kelly JW, Mar VJ. Metastatic pathways in patients with cutaneous melanoma. Pigment Cell Melanoma Res 2017;30:13-27.

3. Hayward NK, Wilmott JS, Waddell N, Johansson PA, Field MA, Nones K, et al. Whole-genome landscapes of major melanoma subtypes. Nature 2017;545:175-80.

4. Alexandrov LB, Nik-Zainal S, Wedge DC, Aparicio SA, Behjati S, Biankin AV, et al. Signatures of mutational processes in human cancer. Nature 2013;500:415-21.

5. Arun G, Diermeier S, Akerman M, Chang KC, Wilkinson JE, Hearn S, et al. Differentiation of mammary tumors and reduction in metastasis upon Malat1 lncRNA loss. Genes Dev 2016;30:34-51.

6. Gupta RA, Shah N, Wang KC, Kim J, Horlings HM, Wong DJ, et al. Long non-coding RNA HOTAIR reprograms chromatin state to promote cancer metastasis. Nature 2010;464:1071-6.

7. He L, Thomson JM, Hemann MT, Hernando-Monge E, Mu $\mathrm{D}$, Goodson $\mathrm{S}$, et al. A microRNA polycistron as a potential human oncogene. Nature 2005;435:828-33.

8. Klein U, Lia M, Crespo M, Siegel R, Shen Q, Mo T, et al. The DLEU2/miR-15a/16-1 cluster controls B cell proliferation and its deletion leads to chronic lymphocytic leukemia. Cancer cell 2010;17:28-40.

9. Memczak S, Jens M, Elefsinioti A, Torti F, Krueger J, Rybak A, et al. Circular RNAs are a large class of animal RNAs with regulatory potency. Nature 2013;495:333-8.

10. Gao J, Xu W, Wang J, Wang K, Li P. The Role and molecular mechanism of non-Coding RNAs in pathological cardiac remodeling. Int J Mol Sci 2017;18:608.

11. Croce CM. Causes and consequences of microRNA dysregulation in cancer. Nat Rev Genet 2009;10:704-14.

12. Chan SH, Wang LH. Regulation of cancer metastasis by microRNAs. J Biomed Sci 2015;22:9.

13. Jing Z, Han W, Sui X, Xie J, Pan H. Interaction of autophagy with microRNAs and their potential therapeutic implications in human cancers. Cancer Lett 2015;356:332-8.

14. Su Y, Li X, Ji W, Sun B, Xu C, Li Z, et al. Small molecule with big role: MicroRNAs in cancer metastatic microenvironments. Cancer Lett 2014;344:147-56.

15. Chan B, Manley J, Lee J, Singh SR: The emerging roles of microRNAs in cancer metabolism. Cancer Lett 2015;356:3018.

16. Ju H, Zhang L, Mao L, Liu S, Xia W, Hu J, et al. Altered expression pattern of circular RNAs in metastatic oral mucosal melanoma. Am J Cancer Res 2018;8:1788-1800.

17. Wang Q, Chen J, Wang A, Sun L, Qian L, Zhou X, et al. Differentially expressed circRNAs in melanocytes and melanoma cells and their effect on cell proliferation and invasion. Oncol Rep 2018;39:1813-24.

18. Yang X, Li Y, Liu Y, Xu X, Wang Y, Yan Y, et al. Novel circular RNA expression profile of uveal melanoma revealed by microarray. Chin J Cancer Res. 2018;30:656-68.

19. Bian D, Wu Y, Song G. Novel circular RNA, hsa_circ_0025039 promotes cell growth, invasion and glucose metabolism in malignant melanoma via the miR-198/CDK4 axis. Biomed Pharmacother 2018;108:165-76.

20. Luan W, Shi Y, Zhou Z, Xia Y, Wang J. circRNA_0084043 promote malignant melanoma progression via miR-153-3p/ Snail axis. Biochem Biophys Res Commun 2018;502:22-9.

21. Shimaoka M, Kawamoto E, Gaowa A, Okamoto T, Park EJ. Connexins and Integrins in Exosomes. Cancers 2019;11:106.

22. Hanniford D, Ulloa-Morales A, Karz A, Berzoti-Coelho MG, Moubarak RS, Sánchez-Sendra B, et al. Epigenetic Silencing of CDR1as Drives IGF2BP3-Mediated Melanoma Invasion and Metastasis. Cancer Cell 2020;37:55-70.

23. Jin C, Dong D, Yang Z, Xia R, Tao S, Piao M, et al. CircMYC Regulates Glycolysis and Cell Proliferation in Melanoma. Cell Biochem Biophys 2020;78:77-88.

24. Zou Y, Wang SS, Wang J, Su HL, Xu JH. CircRNA_0016418 expedites the progression of human skin melanoma via miR625/YY1 axis. Eur Rev Med Pharmacol Sci 2019;23:1091830.

25. Lin Q, Jiang H, Lin D. Circular RNA ITCH downregulates GLUT1 and suppresses glucose uptake in melanoma to inhibit cancer cell proliferation. J Dermatolog Treat 2019:1-5.

26. Zhang L, Li Y, Liu W, Li H, Zhu Z. Analysis of the complex interaction of CDR1as-miRNA-protein and detection of its novel role in melanoma. Oncol Lett 2018;16:1219-1225.

27. Shang Q, Li Y, Wang H, Ge S, Jia R. Altered expression profile of circular RNAs in conjunctival melanoma. Epigenomics 2019;11:787-804.

28. Chen Z, Chen J, Wa Q, He M, Wang X, Zhou J, et al. Knockdown of circ_0084043 suppresses the development of human melanoma cells through miR-429/tribbles homolog 2 axis and Wnt/beta-catenin pathway. Life Sci 2020;243:117323.

This is an open access article distributed under the terms of the Creative Commons Attribution-NonCommercial-ShareAlike 3.0 License, which allows others to remix, tweak, and build upon the work non-commercially, as long as the author is credited and the new creations are licensed under the identical terms

This article was originally published in a special issue, "Biomedical research applications in Pharmaceutical Sciences" Indian J Pharm Sci 2020:82(2)spl issue2;45-48 